\title{
Developmental Anomalies of Temporal Muscle
}

\section{Jorge Orlando Guerrissi and Gustavo Gabriel Cotroneo*}

Hospital cosme argerich, ciudad de buenos aires, buenos aires, Argentina

*Correspondimg author: Gustavo Gabriel Cotroneo, Hospital cosme argerich plastic and reconstructive surgery, pyy margal 750, ciudad de buenos aires, buenos aires C1155AHD, Argentina, Tel: +5491132266671/41210900; E-mail: cotro_@hotmail.com

Rec date: May 21, 2014; Acc date: Jul 7, 2014; Pub date: Jul 9, 2014

Copyright: (C) 2014 Guerrissi JO, et al. This is an open-access article distributed under the terms of the Creative Commons Attribution License, which permits unrestricted use, distribution, and reproduction in any medium, provided the original author and source are credited.

\begin{abstract}
The anatomy of the temporal region is complex. Also there is controversy over the structures that make up the region.

Thus than classically described anatomical structures as fascias, temporal muscle, frontal nerve, arteries, the superficial temporal muscle is present in a variable number of cases.

This muscle represents fibrous regression of named superficial temporal muscle very developed in animals with a specific masticatory action. In human has no physiological importance but its knowledge is important for plastic and maxillofacial surgeons to undertake surgeries in the region since it can generate confusion over anatomical planes and their relationship to vessels and nerves.
\end{abstract}

Keywords: Developmental anomalies; Superficial temporal muscle; Temporoparietal fascia; Temporal region

\section{Introduction}

The temporal region has anatomical structure of subcutaneous fascias that confuses not only the constitution of the region but also during plastic and reconstructive surgical maneuvers. Identify different fascias and its connections with numerous nerve and artery branches that cross the region is especially important for plastic surgeons.

The first fascial layer of the region is called temporoparietal fascia and it is located below the hair follicles and subcutaneous tissue of the temporoparietal region. It is considered a cephalic extension of the superfical musculoaponeurotic system described by Mitz $\mathrm{V}$ and Peyronie M (1974) and continues in all directions with other structures of this system [1].

Anteriorly, it is continuous with the frontalis and orbicularis oculi muscle, whereas posteriorly, it blends with the occipitalis and posterior auricular muscles. Superiorly, the fascia merges with the galea aponeurotica and inferiorly it is continuous with the superficial musculoaponeurotic system $[2,3]$.

Traditionally, it has been described as a single sheet, although other authors such as Knize DM [4] and Tellioğlu AT [5] mention that this would comprise two sheets. Histological studies showed the presence of a thin muscular sheet in the outer sheet of the temporoparietal fascia below the temporal line.

This muscle corresponds to the superficial temporal muscle present in some animals but that it has been transform rudimentary in humans and only remains superficially covering the temporal region. These muscle fibers lack functionality and constitute only anatomical finding.
A loose areolar tissue plane lies deep to the temporoparietal fascia and extends beneath the entire superficial fascia system of the scalp, including the galea aponeurotica and the frontalis and occipitalis muscle.

Deeper is the temporal fascia, which surrounds the temporal muscle. Underneath the muscle, this fascia merges with the periosteum of the temporal, frontal and parietal bone. Superiorly, the temporal fascia inserts in the superior temporal line and inferiorly it inserts in the zigmatic arch. [6].

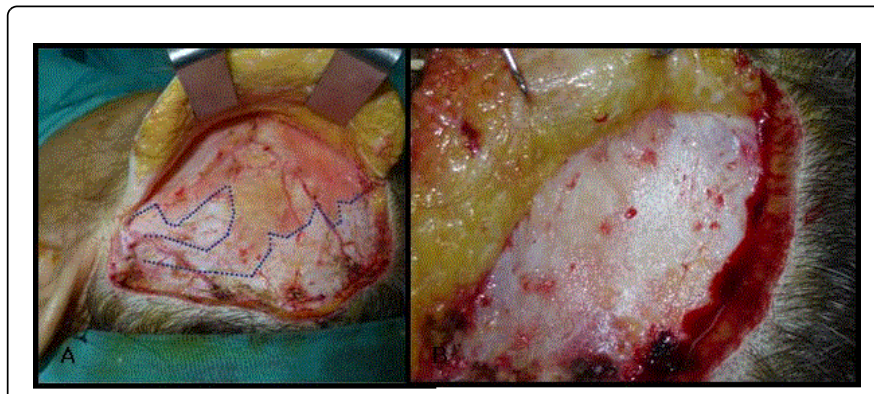

Figure 1: Temporal fascia

There is no doubt that the most important anatomic element in the region is the frontal branch of the facial nerve.

The facial nerve runs almost horizontally to the parotid $2 \mathrm{~cm}$ below the zygomatic arch, heading obliquely from back to front, from inside to outside and from top to bottom. As it leads to the periphery of the parotid gland, it becomes more superficial. Inside of the parotid gland, the division of the primary branches occurs: an upper, the temporofacial and lower the cervical facial [7].

When the temporo-facial branch reaches the level of the mandibular condyle, it is divided into several secondary trunks, which usually anastomose forming true plexuses. The branches that arise 
from the temporo-facial trunk are: 1 . Frontal; 2. Temporal; 3. Eyelid branches; 4. Zigomatics and 5. Upper mouth.

Regarding the temporal region above the zygomatic arch, the frontal branch is located in the existing plane between the temporoparietal fascia and the superficial layer of the temporal fascia.

In the cases where the superficial temporal muscle is present, there are no changes in the ordinary relationships of the facial nerve and vassels since this rudimentary muscle stays in a more superficial plane just below hair folicules.

This paper has following objective to clarify anatomical knowledge of the temporoparietal region, principally when fibers of temporal superficial muscle are presents avoiding erroneous surgical maneuvers and potentially dangerous for both regional nerves and vessels.

\section{Materials and Methods}

It is difficult to establish the true incidence rate of this muscle abnormality, but In 58 surgeries performed in the temporal region in the Plastic and Reconstructive Department of Cosme Argerich Hospital in Buenos Aires was evident the presence of superficial muscle fibers confirmed by direct visualization and histologic studies in 29 patients. The histological study confirmed striated skeletal muscle with hematoxilin and eosin staining.

Twenty patients (69\%) were operated of aging face by mean of superficial and submuscular lifting and other 9 (31\%) underwent maxillofacial surgeries in TMJ, superior maxilla and zigomatic arch.

During undermining of temporal area, atrophic muscular fibers were found immediately below subcutaneous fat layer into temporoparietal fascia. In all cases ( 29 patients) muscle fibers were very thin, forming isolated groups extended on temporal area.

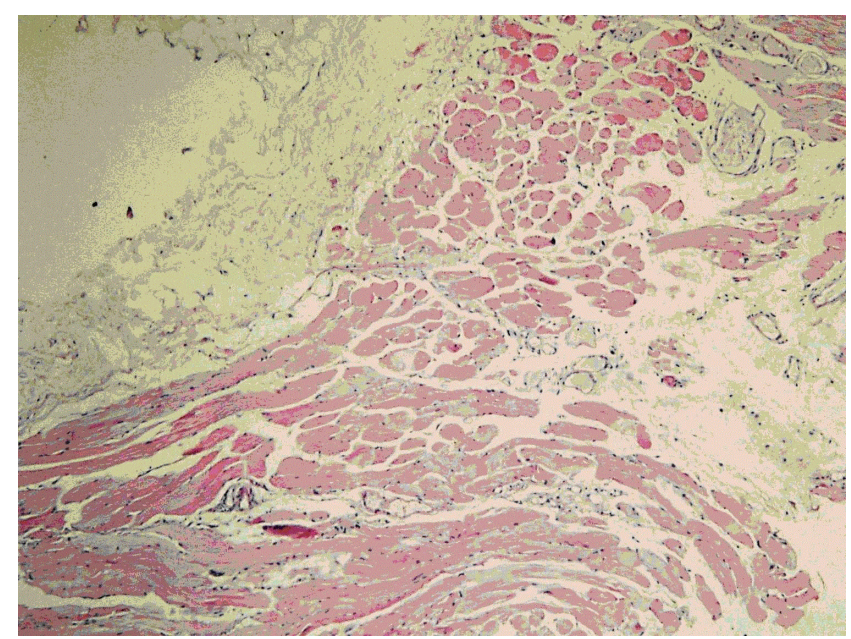

Figure 2: Striated skeletal muscle with hematoxylin and eosin staining

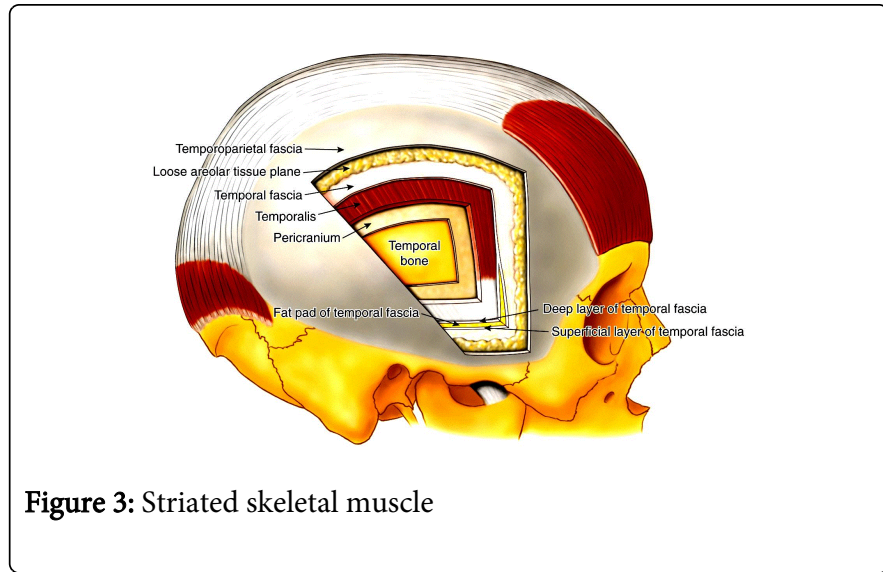

\section{Superficial Temporal Muscle and Comparative Anatomy}

Oxnard et al. in their research note 400 human corpses, 35 of which possessed the superficial temporal muscle. These bodies possessing the superficial temporal muscle were dissected. Furthermore dissected 4 chimpanzes, 4 rhesus monkeys, colobus monkeys and other species [8].

In monkeys and apes, the arrangement of the dissected temporal muscles at work is consistent with primate anatomy texts. A superficial fleshy head of the temporalis muscle takes origin from the skull area between the superior and inferior temporal ridges, from the ridges themselves and to a slight degree from the outer surface of the underlying deep head. Further a few aditional fibres taking origin from the internal surface of the zygomatic arch. This superficial muscle is covered by deep fascia which arises from the superior temporal ridge of the skull and passes downwards on the surface of the superficial fibres of the muscle to the coronoid process of the mandible.

The main (deep) part of the temporal muscle is below the superficial temporal muscle and arises from the periosteum covering a large part of the lateral surface of the skull below the inferior temporal ridge. This muscle gives way to a glistening silver tendon which also inserts on the coronoid process of the mandible. This muscle is covered by deep fascia which arises, as does the deep fascia lining the undersurface of the superficial head, from the inferior temporal ridge. The fact, however, that these two layers are separate implies that, at least on occasion, these two muscles are capable of contracting independently, even thought they might often act together.

In humans, the temporalis muscle is different. It arises from the cranium at and below the inferior temporal line (a thin line in humans, rather a strong ridge as in apes and most large monkeys). It is characterised by a glistening silver tendon just like that of the deep head in apes and monkeys. Underlying the human temporalis muscle is a deeper layer of deep fascia entirely similar to the deepest layer under the deep head in apes and monkeys.

Oxnard CE in his research dissected 35 cadavers in each of which a complete or partial superficial head of the temporalis muscle was present. These anomalous muscular heads extends from situations just like in apes and monkeys where the entire muscle was present, though very much thinner, to situations in which lesser portions of the muscle were found. 
There were 4 cases (1\%) with a superficial head entirely similar, though very much thinner, to that in apes and monkeys. There were 31 cases $(8 \%)$ in which a partial superficial head was present. This muscle arises from the area comprised between the temporal lines. It has a cranial part thay may have muscle fibers or have mostly aponeurotic component. As in apes and monkeys, this muscle receives muscular fibers from the surface of the deep temporal muscle and the internal face of the zygomatic arch and directed to the coronoid process of mandible [8].

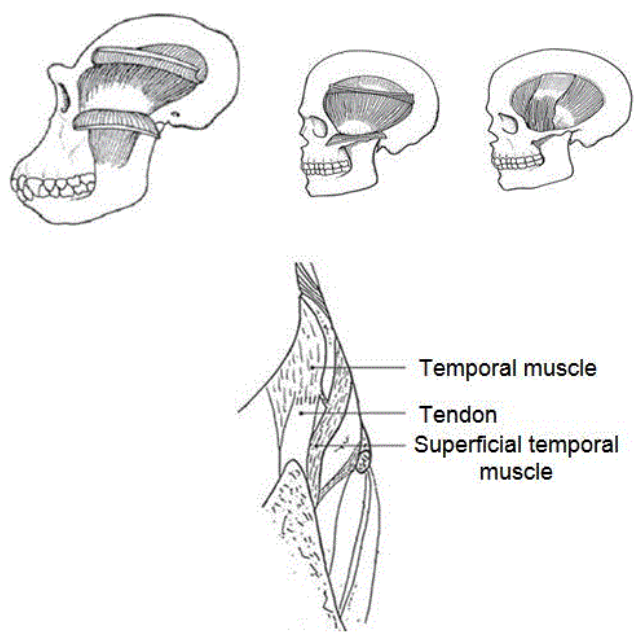

Figure 4: Temporal muscle

Why the loss of this temporal muscle has occurred in humans? An obvious possibility is that changed masticatory habits and mechanics did not render such reduction or loss deleteious, whereas such changes would be inmediately eliminated in creatures with the diets of most apes and monkeys.

Furthermore, Testut, in his description of the epicranial fascia or aponeurotic galea expressed that morphologically the galea should be considered, as the muscles that attaches, as a portion of the panniculus carnosus, which originally muscular, has experienced during its development a fibrous regression $[9,10]$.

\section{Discussion}

The temporal region is a complex anatomical area due to its composition of multiple layers and there is a strong semantic and practical controversy over its component structures and the relationships they have with each other.

Understanding the anatomy of the area is important for the plastic and head and neck surgeons, to accurately identify different fascias that span the region and its connections with numerous nerve and artery branches that cross the region. It is essential to know the frontal branch of the temporo-facial branch of the facial nerve that extends obliquely from the zygomatic arch to reach the deepest part of the frontal muscle.

The preservation of axonal integrity is the primary care should have the plastic surgeon in the dissection of the temporal region for aesthetic or reconstructive surgeries.
The presence of muscle fibers in superficial planes may confuse the surgeon who can work in a wrong plane and also, he can make future surgical maneuvers that may endanger any of the neural structures mentioned.

There is sufficient evidence to show the presence of a thin muscular layer or even isolated muscle fibers in the temporoparietal fascia, below the hair follicles.

This muscle corresponds to the superficial temporal muscle present in animals that has been devolving in humans, but there may be remains superficially covering the temporal region; these muscle fibers lack functionality and are only anatomical finding.

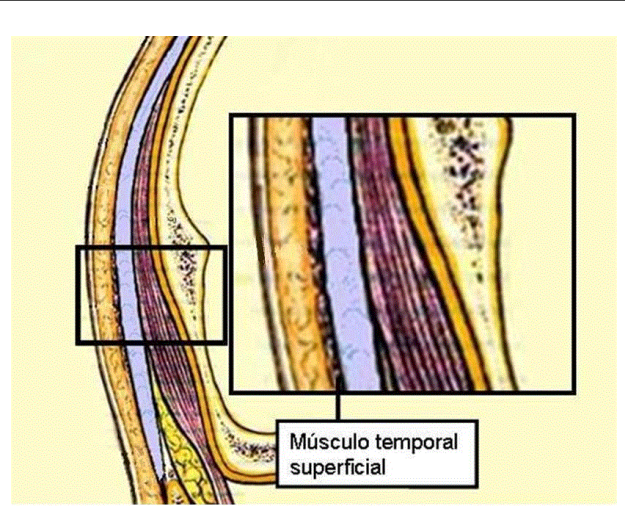

Figure 5: Superficial temporal muscle

Of the different explanatory theories about the persistence of this muscle, have value two: 1) remnant of the superficial temporal muscle and 2) remaining panniculus carnosus

Animals including lower mammals, have muscles that are attached to the skin, these are called skin muscles or panniculus carnosus [11]. These muscles allow the animal to mobilize certain areas of the skin, apparently as a protective measure to ward located noxas agents, such as insects. In human limbs have evolved so much that can reach any part of the body. For this reason, the panniculus carnosus became obsolete and has devolved. But remnants of it can be found in some individuals. Some muscles may contain remnants of the panniculus carnosus as the pectoralis major, trapezius, serratus, pyramidalis, palmaris longus and some craniofacial muscles.

\section{Conclusion}

It is essential the knowledge of the complex anatomy of the temporal region for the surgeon that undertake any surgery in the region. Fibers of the superficial temporal muscle can be usually finding; though they have no functional or physiological significance but surgical importance.

From another point of view, the presence of this muscle must be known by maxillofacial surgeons when they perform surgeries on the region in case of both superior mandible or TMJ approaches.

For the plastic surgeon also it is important to know the existence of the superficial temporal muscle and that may become apparent during the regional dissection in the treatment of periorbital aging or any other variety of rhytidectomies confusing the surgeon about the exact location of the anatomical planes and their relation to nerve and vascular structures. 
Citation: Guerrissi JO, Cotroneo GG (2014) Developmental Anomalies of Temporal Muscle. Surgery Curr Res 4: 199. doi:

Page 4 of 4

\section{References}

1. Mitz V, Peyronie M (1976) The superficial musculo-aponeurotic system (SMAS) in the parotid and cheek area. Plast Reconstr Surg 58: 80-88.

2. Accioli de Vasconcellos JJ, Britto JA, Henin D, Vacher C (2003) The fascial planes of the temple and face: an en-bloc anatomical study and a plea for consistency. Br J Plast Surg 56: 623-629.

3. Krayenbuhl N, Isolan G, Hafez A, Ysargil M (2007) The relationship of the frontotemporal branches of the facial nerve to the fascias of the temporal region: a literature review applied to practical anatomical dissection. Neurosurg Rev 30: 8-15.

4. TellioÄŸlu AT, Tekdemir I, Erdemli EA, Tüccar E, Ulusoy G (2000) Temporoparietal fascia: an anatomic and histologic reinvestigation with new potential clinical applications. Plast Reconstr Surg 105: 40-45.

5. Knize DM (1996) An anatomically based study of the mechanism of eyebrow ptosis. Plast Reconstr Surg 97: 1321-1333.
6. Davidge KM, Van Furth W, Agur A (2010) Naming the soft tissue layers of the temporoparietal region: unifying anatomic terminology across surgical disciplines. Neurosurgery 67: 120-130.

7. Guerrissi Jorge Orlando y col (2007) Cirugía de los tumores de la glándula parótida. Buenos Aires, AMOLCA.

8. Oxnard CE, Franklin D (2008) Ghosts of the past I: some muscles and fasciae in the head domain. Folia Primatol (Basel) 79: 429-440.

9. Testut L, Latarjet A (1951) Tratado de anatomía humana. Salvat. (9th edn).

10. Testut L (1884) Les anomalies musculares chez l'homme. Masson G. Paris

11. Stevenson R, Hall J (2006) Human malformations and related anomalies. Oxford University Press: 800-801. 